SESSION V. MASS LOSS - Chair: Lindsey F. Smith 


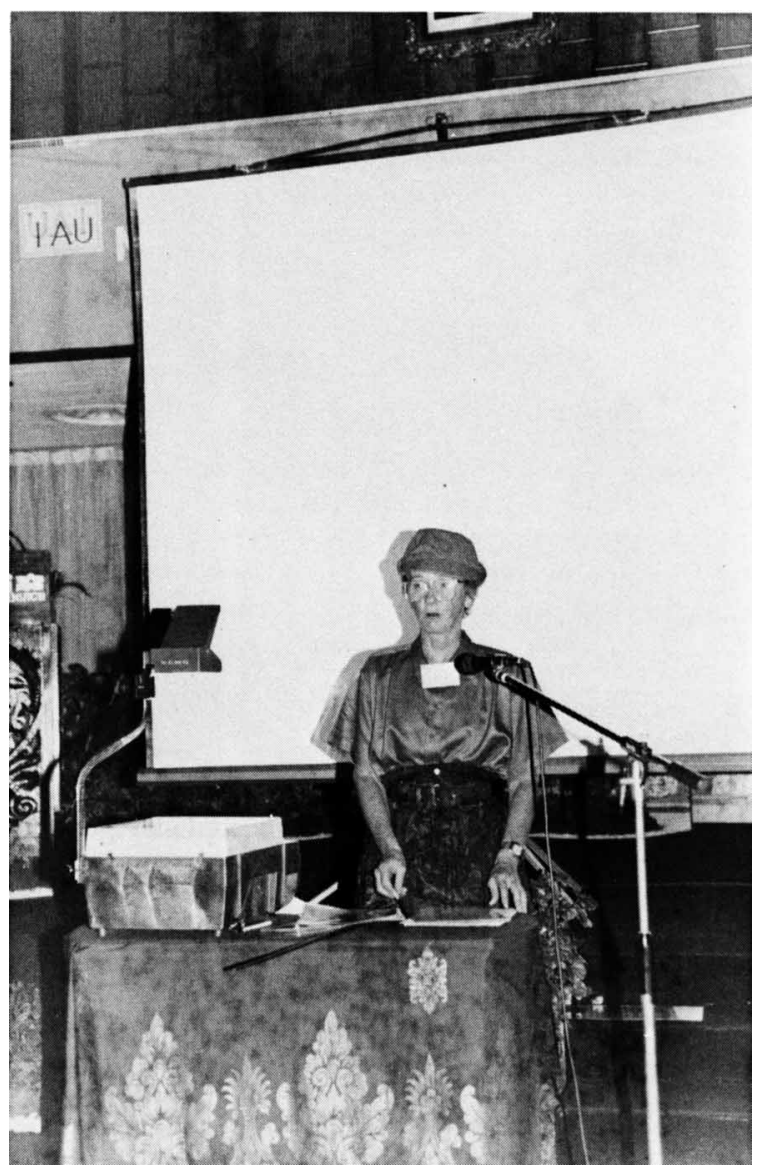

Lindsey Smith 


\title{
OBSERVATIONS OF WOLF-RAYET MASS LOSS
}

\author{
Allan J Willis \\ Department of Physics \& Astronomy \\ University College London \\ Gower Street \\ London WC1E 6BT, UK
}

\begin{abstract}
Current knowledge of the stellar winds and mass loss rates for WR stars is reviewed. Recent IR spectroscopy and reassessments of UV resonance line P Cygni profiles have led to revisions of terminal velocities, with $\mathrm{v}_{\infty} \simeq 0.75 \times$ previous estimates. Radio and IR $\left(10_{\mu m}\right)$ free-free emission for well-established thermal sources, coupled with recent considerations of the wind ionisation balance and chemistry, leads to WR mass loss rates lying in the range $10^{-5}-10^{-4} \mathrm{M}_{\odot} \mathrm{yr}^{-1}$. This scale is confirmed by independent analyses of optical polarisation modulation in $\mathrm{WR}+\mathrm{O}$ binaries. No significant differences are apparent between the mean mass loss rates of: (a) single and binary WR stars; (b) WN and WC stars, and (c) the WN and WC subclasses. The overall mean WR mass loss rate is $\sim 5 \times 10^{-5} \mathrm{M}_{\odot} \mathrm{yr}^{-1}$. Although WR radiative luminosities are uncertain, there may be a rough scaling of $\dot{M}_{W R}$ with $\mathrm{L}_{*}$, with a spread of up to an order of magnitude at a given $\mathrm{L}_{*}$. WR winds have the highest momenta of the hot luminous stars, with values of $\dot{M} \mathrm{v}_{\infty} \mathrm{c} / \mathrm{L}_{*}$ in the range 1-30 (WN7,8 and WC9 stars may lie near the lower bound). An additional mechanism to radiation pressure may be required to initiate the high WR mass loss, although thereafter the winds may be radiatively accelerated. Intrinsic variability in optical light, polarisation and emission lines, and in UV P Cygni profiles, indicate significant instability in the WR winds. For extragalactic WR stars in the Local Group, optical line strengths and widths do not suggest substantial differences in wind velocities and mass loss rates of subtypes compared to galactic counterparts.
\end{abstract}

\section{Introduction}

It is well established that the predominant emission line spectrum of the WR stars is a direct reflection of their high levels of mass loss (Abbott \& Conti 1987). Characterising their stellar winds and mass loss rates as a function of subtype is thus important in developing a quantitative understanding of their physical and chemical properties and evolutionary status from both observational and theoretical standpoints. This paper reviews progress in observational studies of WR mass loss and stellar winds, emphasising recent work over the past five years or so. I shall not dwell much on the theory of WR mass loss - this will be covered in the next review, by Cassinelli (these proceedings). Excellent comprehensive reviews of earlier work in this field have been given by Barlow (1982) and in the exemplary paper by Abbott et al. (1986). 


\section{Radio and IR continuum data}

Abbott et al. (1986, hereafter ABCT) provide the most extensive set of $4.9 \mathrm{GHz}$ radio observations of WR stars from their own data and earlier work by Hogg (1982, 1985), Dickel et al. (1980), Florkowski (1982) and Becker \& White (1985). In their sample 22 stars have measured $4.9 \mathrm{GHz}$ fluxes, whilst upper limits were obtained for a further 13 stars. ABCT also present radio observations at $14.9 \mathrm{GHz}$ for 6 stars based on their data and that of Hogg (1982). The radio flux is presumed to arise from free-free emission in the outermost regions of the WR winds, where the wind velocity has reached its terminal value, $\mathbf{v}_{\infty}$. In spherical symmetry, the formula developed by Wright \& Barlow (1975) may then be used to deduce the rate of mass loss, viz.:

$$
\dot{M}=0.095 v_{\infty}\left(\frac{S_{\nu}^{0.75} D^{1.5}}{(g \nu)^{0.5}}\right)\left(\frac{\mu}{Z \gamma^{0.5}}\right) \quad M_{\odot \mathrm{yr}^{-1}}
$$

where $S_{\nu}$ is the radio flux in Jy at frequency $\nu$ in $\mathrm{Hz} ; v_{\infty}$ in $\mathrm{km} \mathrm{s}^{-1} ; D$ is the distance in kpc; $g$ is the gaunt factor; $\mu, \mathrm{Z}$ and $\gamma$ are respectively the mean molecular weight, r.m.s. ionic charge and mean number of electrons per ion. With $X_{i}, M_{i}$ and $Z_{i}$ as the fractional abundance, molecular weight and ionic charge of species $i$, then:

$$
\mu=\frac{\sum X_{i} M_{i}}{\sum X_{i}} ; \quad Z=\frac{\left(\sum X_{i} Z_{i}^{2}\right)^{0.5}}{\sum X_{i}} ; \quad \gamma=\frac{\sum X_{i} Z_{i}}{\sum X_{i}}
$$

In the radio region, the free-free spectrum is predicted to have a distribution $\mathrm{S}_{\nu} \propto \nu^{\alpha}$, with $\alpha=0.6$, (the departure in $\alpha$ from a value of $2 / 3$ reflects the frequency dependence of the gaunt factor). ABCT considered three methods to test the validity of interpreting the radio data as thermal, free-free emission: (i) interferometric observations of the visibility function compared to predictions of thermal wind models, (ii) consideration of the observed radio or radio-IR spectral index, and (iii) comparison of the observed radio flux with that predicted from the observed optical recombination line emission measure.

Hogg (1985) has used the VLA to resolve the radio emission of $\gamma$ Velorum at $1.49 \mathrm{GHz}$, 4.9 GHz and 14.9 GHz (see Fig 1), confirming a thermal origin consistent with spherical outflow. The frequency dependence of the visibility functions requires an electron density decrease more rapid than $\mathrm{r}^{-2}$ for wind distances greater than $3 \times 10^{15} \mathrm{~cm}$ which is commensurate with helium (the dominant electron source) beginning to recombine. A mass loss rate of $8.6 \pm 1.0 \times 10^{-5} \mathrm{M}_{\odot} \mathrm{yr}^{-1}$ and electron temperature of $5600 \pm 500 \mathrm{~K}$ provided the best fit to the multifrequency radio interferometric data.

Five of the six stars listed by ABCT with 4.9 and $14.9 \mathrm{GHz}$ radio data (but generally too faint to determine visibility functions) show a radio spectral indedx of $\alpha \sim 0.6$, confirming their emission to be thermal (the sixth star, WR 125, has a measured index of -0.5 and is prescribed as definitely non-thermal in nature). For $\gamma$ Velorum they confirm the result from Purton et al. (1982) who deduced $\alpha=0.58 \pm 0.17$ from data at 5.0, 6.2, 8.1, and $14.5 \mathrm{GHz}$.

Williams et al. (1990) present observations between $1.25 \mu \mathrm{m}$ and $1100 \mu \mathrm{m}$ of $\gamma$ Velorum, combined with the radio measurements by Hogg (1982) at $4.9 \mathrm{GHz}$ and by Jones (1985) at $0.843 \mathrm{GHz}$ to examine the spectral distribution of the wind emission (Fig 2). They find $\alpha=0.69 \pm 0.02$ between the $1100 \mu \mathrm{m}$ and radio wavelengths, which is consistent with plausible variations of temperature and ionisation in the wind.

Barlow, Smith \& Willis (1981, hereafter BSW) present infrared $10 \mu \mathrm{m}$ fluxes for 21 galactic WR stars corrected for the underlying photospheric contribution to estimate the excess wind emission at this wavelength. The $10 \mu \mathrm{m}$ excess emission data from BSW for 12 
Fig 1: Radio emission of $\gamma$ Vel resolved with the VLA (from Hogg 1985)

Fig 2: The optical-IR-mm-radio emission of $\gamma$ Vel (from Williams et al. 1990).
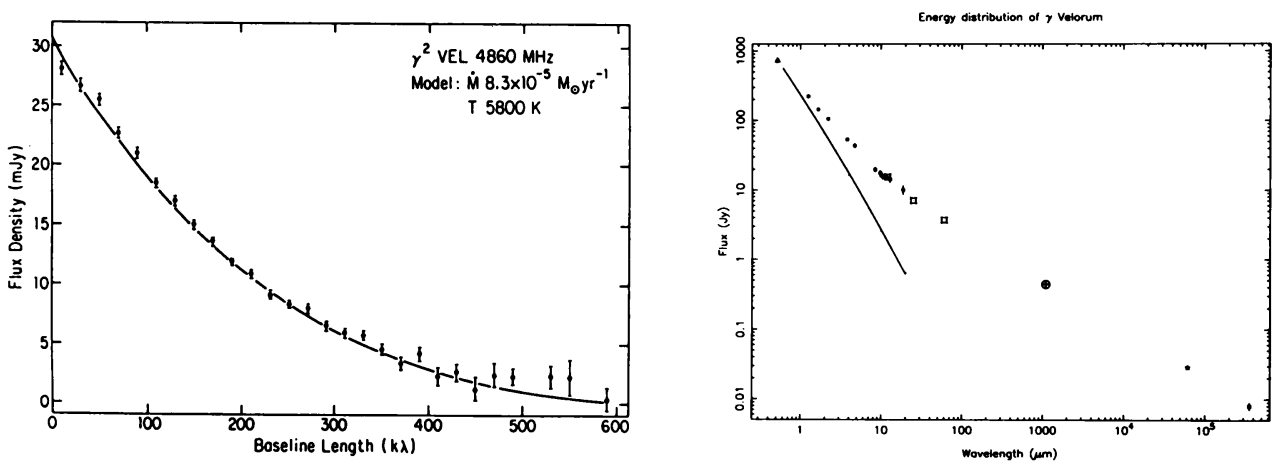

stars with measured $4.9 \mathrm{GHz}$ radio fluxes give IR-radio spectral indices in the tight range of $0.66-0.82$ (see Table 1). HD 193576 exhibits a $10 \mu \mathrm{m}-4.9 \mathrm{GHz}$ index of 0.53 - identical to that expected for a constant velocity isothermal flow at both wavelengths. The mean value (HD 193576 omitted) of $\alpha=0.75 \pm 0.04$ is similar to the mm-radio spectral index determined by Williams et al. for $\gamma$ Velorum (a confirmed thermal source), suggesting that such IR-radio indices also indicate thermal emission (with the difference from 0.53 reflecting changing temperature and/or ionisation conditions in the IR and radio emitting regions).

Table 1 lists the $4.9 \mathrm{GHz}$ fluxes for stars which ABCT have confirmed as thermal emitters using one or more of the above considerations, together with the $10 \mu \mathrm{m}$ data from BSW, including stars with IR but no radio measurements. For such cases, the radio flux can be predicted using the $10 \mu \mathrm{m}$-radio index of 0.75 (BSW). In $\S 5$, these data are combined with reassessments of wind terminal velocities, chemistry and ionisation balance to update the mass loss rate scale for WR stars.

\section{Terminal velocities and $\mathbf{v}(\mathbf{r})$}

Until recently (as for analyses of OB mass loss), $\mathbf{v}_{\infty}$ has been taken as the maximum edge velocity of the $\mathrm{P}$ Cygni absorption components in the UV resonance lines (e.g. Willis 1982), or deduced from the maximum velocity inferred from the widths of optical emission lines (e.g. for WN stars from Conti Leep \& Perry 1983, or for WC stars from Torres, Conti \& Massey 1986 who extrapolate the emission line width vs. excitation potential correlations to zero E.P. to predict $\mathrm{v}_{\infty}$ ). ABCT used these approaches to derive values of $v_{\infty}$ for the stars in their radio sample noting the good agreement between the optical results and the edge velocities of the UV P Cygni line profiles for stars in common. These approaches in effect estimate the maximum velocities which can be associated with any individual WR stellar wind material, $\mathbf{v}_{\max }$, which are listed in Table 1 . Several recent papers have questioned whether these values of $v_{\max }$ are to be identified with the true terminal velocity of the wind associated with the bulk material flow, and suggested lower values of $\mathbf{v}_{\infty}$.

Williams \& Eenens (1989) measured the P Cygni absorption velocities in the He I 2.058 $\mu \mathrm{m}$ line in $8 \mathrm{WR}$ stars finding values $\sim 0.7$ of the terminal velocities deduced from the 
Fig 3: The CIV $\lambda 1550 P$ Cyg profile in $H D 165763$ (from $P B H$ )

Fig 4: $v_{\infty}$ vs. WR subtype - filled symbols $=v_{\text {black }}(P B H)$; open symbols $=0.74 v_{\max }$
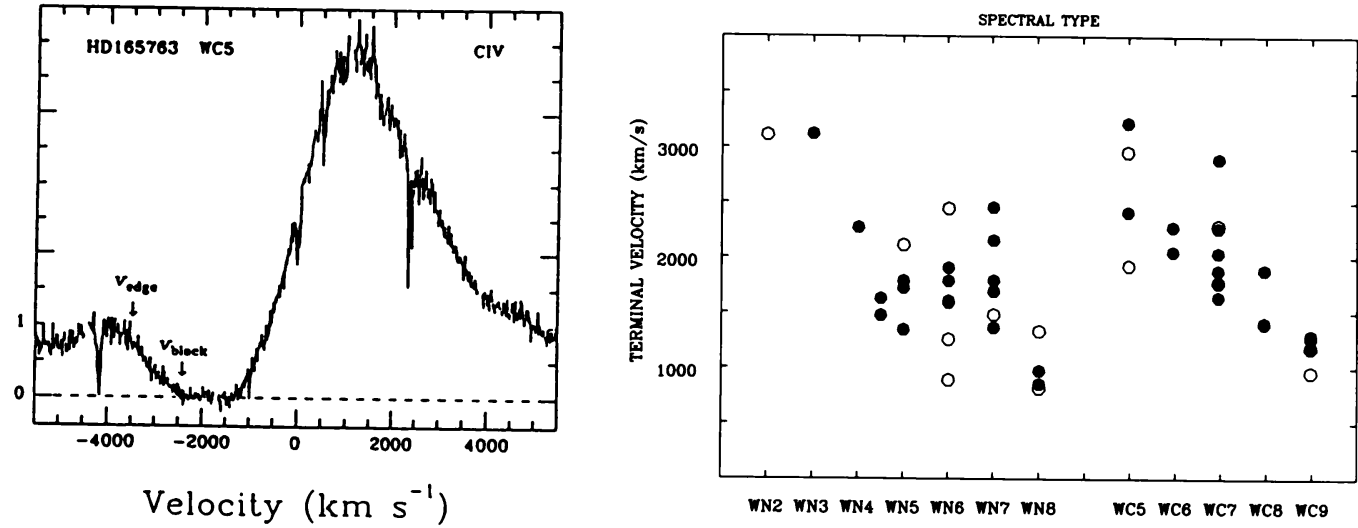

$\mathbf{v}_{\max }$ methods discussed above. They argue that this HeI line is formed in the outermost regions of the WR winds and thus provides more suitable measurements of $\mathrm{v}_{\infty}$. Barlow, Roche \& Aitken (1988) in their analysis of $12-13 \mu \mathrm{m}$ spectra of $\gamma$ Velorum, measured a terminal velocity from the [Ne II] $12.8 \mu \mathrm{m}$ forbidden line of $1520 \mathrm{~km} \mathrm{~s}^{-1}$, considerably lower than the value of $\sim 2000 \mathrm{~km} \mathrm{~s}^{-1}$ deduced previously from the UV P Cygni profiles. The [Ne II] line exhibits a rectangular profile as expected for an optically thin line formed at large radii and constant velocity, and the typical density for its formation of $5 \times 10^{5}$ $\mathrm{cm}^{-3}$ suggesting an emission region radius of $\sim 1300 \mathrm{R}_{*}$. The HeI $2.058 \mu \mathrm{m}$ line in $\gamma$ Velorum shows a P Cygni central absorption at $\sim 1500 \mathrm{~km} \mathrm{~s}^{-1}$, in accord with the [Ne II] data suggesting that the measurements given by Williams and Eenens (1989) do indeed reflect the true $W R$ wind terminal velocities.

Recently, Prinja, Barlow \& Howarth (1990, hereafter PBH) have reassessed inferences on $\mathrm{v}_{\infty}$ from UV $\mathbf{P}$ Cygni absorption profile measurements of both OB and WR spectra. They point out (as others have in the past) that the UV resonance line $P$ Cygni saturated profiles generally show extended regions of zero residual intensity (up to some maximum velocity, vblack) and additional (non-black) absorption extending to higher velocities up to the maximum value observed, $v_{\text {edge }}$ (Fig 3 ). The observed profiles differ significantly from those expected in Sobolev models of $\mathrm{P}$ Cygni line formation with monotonic velocity laws, where zero residual intensity is only reached at $\mathbf{v}_{\infty}$ and the profile should then rise immediately to the continuum. These differences are now generally explained in terms of non-monotonic wind velocity laws and shocks in the winds induced by instabilities in the flows (Lucy 1982, 1983, Owocki, Castor and Rybicki 1988), with $\mathbf{v}_{\text {edge }}-\mathrm{v}_{\text {black }}$ a measure of the velocity amplitude of the shocks. PBH argue that $v_{\text {black }}$ is a more meaningful measurement of $\mathrm{v}_{\infty}$ and present values for $35 \mathrm{WR}$ stars with available high resolution IUE spectra, finding a mean ratio of $\mathrm{v}_{\infty} / \mathrm{v}_{\text {edge }}=0.76 \pm 0.12$. A subset of their data is given in Table 1. For 15 stars in common with the data of $\mathrm{PBH}$ and $\mathrm{ABCT}$ the mean ratio of $\mathrm{v}_{\text {black }} / \mathrm{v}_{\max }=0.74 \pm 0.08$, and I have adopted this scaling factor for those stars with radio data but no $\mathrm{v}_{\text {black }}$ measurement to estimate appropriate values of $\mathrm{v}_{\infty}$ in deducing mass loss rates (see below).

Fig 4 plots these revised values of $v_{\infty}$ vs. spectral type for both galactic WN and WC stars. For the WC stars there is a clear trend of increasing $\mathbf{v}_{\infty}$ with earlier subtype, in keeping with the well known correlation of optical line width with subclass (see Torres et al. 1986). For the WN stars, no clear trend is apparent, although the WN8 stars exhibit 
low velocity winds, whilst the very earliest types (WN2,3) have the largest values. Most WN subtypes can show a large range of wind velocities.

Little is known directly about the velocity laws, $\mathrm{v}(\mathrm{r})$, of WR winds. Cherepaschuk et al. (1984) have modelled the IR-UV eclipse data of V444 Cyg to empirically determine the velocity law of the WN5 component wind. Their result is reasonably well fitted with the usual paramaterised law of $\mathbf{v}(\mathbf{r})=\mathrm{v}_{\infty}\left(1-\mathrm{r}_{*} / \mathbf{r}\right)^{\beta}$ with $\beta \sim 1$. This law has generally been adopted in detailed model atmosphere analyses of WR spectra by Schmutz et al. (1988) and Hillier $(1987,1989)$ who are able to reproduce the observed emission line profiles fairly well. For OB stars, Friend \& Abbott (1986) and Pauldrach et al. (1986) include the effects of rotation and finite disk of the star and conclude that a value $\beta \sim 0.8$ in the above velocity law provides the best fit to observational data. Thus it may be that the WR winds, like those for OB stars, are accelerated through radiation pressure, although the very high levels of WR mass loss may require an additional mechanism(s) (see below).

\section{Ionisation structure}

Detailed modelling of the atmospheres of WN and WC stars by Hillier $(1987,1989)$ characterises the ionisation structure of their winds, showing that even for high core temperature models (e.g. with $\mathrm{T}_{\text {eff }}=60,000 \mathrm{~K}$ ) the wind electron temperature can be low (e.g. $20000 \mathrm{~K}$ at $\mathrm{N} e \sim 10^{11}$ and $\sim 9000 \mathrm{~K}$ at $\mathrm{N}_{e} \sim 10^{9}$ ) giving values of $\mathrm{T}_{e}$ in the radio emitting region comparable to that deduced by Hogg (1985) for $\gamma$ Velorum. The ionisation stratification in the Hillier models is compatable with observed correlations of optical line width vs. ionisation potential (e.g. Kuhi 1973). Willis (1982) argued that when the UV resonance and low excitation lines were included with optical measurements, line widths correlated better with excitation potential (E.P.) than with I.P., questioning such an ionisation stratification. As discussed by Hillier (1989) this discrepancy may now be understood, since the UV lines, with their increased opacity, may be tapping lower density, higher velocity material associated with shocked gas in the wind, and should not necessarily be considered together with the optical lines.

In the Wright \& Barlow formulation the factor ' $C$ ' $=\left(\mu /\left(\mathrm{Z} \gamma^{0.5}\right)\right.$ depends upon the chemistry and ionisation balance presumed for the radio-emitting regions, and recent considerations of this parameter have led to revisions in $\dot{M}$.

ABCT assumed only $\mathrm{H}$ and $\mathrm{He}$ ions contributed towards the $C$-factor, and for WN stars estimated the $\mathrm{H}^{+} / \mathrm{He}^{++}$ratio using the Pickering decrement measurements of Conti, Leep \& Perry (1983) and the $\mathrm{He}^{+} / \mathrm{He}^{++}$ratio from measured values of the emission measures of optical He I and He II lines. For WC stars the $\mathrm{H} / \mathrm{He}$ ratio was presumed to be zero, and the $\mathrm{He}$ ionisation balance again inferred from the optical $\mathrm{He} \mathrm{I}$ and $\mathrm{He}$ II emission measures. For most stars $\mathrm{He}^{++}$was taken as the dominant ion in the radio region. However, Schmutz, Hamann \& Wessolowski (1989) conclude that $\mathrm{He}^{+}$is the dominant ion for the great majority of WN and WC subclasses (only three WN2 and WN3 stars in their sample have $\mathrm{He}^{++}$dominant) leading to an upward revision to mass loss rates deduced from the radio data. Van der Hucht, Cassinelli \& Williams (1986) for WC stars included the effects of high $\mathrm{C}$-abundances inferred from evolutionary models together with calculated ionisation equilibria in simplified model atmospheres, to propose an upward revision to the mass loss rates of ABCT of about a factor of 2 for WCE stars and by about a factor of 2-3 for WCL stars.

The present view is that $\mathrm{He}^{+}$is the dominant helium ion in the radio emitting regions for both WN and WC stars. For WNE stars, with $\mathrm{H}^{+} / \mathrm{He}^{++}$generally $\leq 0.2-0.3$ by number (Conti, Leep \& Perry 1983), the effect of $\mathrm{H}$ on the $C$-value is negligible, and thus for the radio-emitting regions we can adopt $\mu=4.0, \mathrm{Z}=1.0$ and $\gamma=1.0$, viz $C=4.0$. For WN7 and WN8 stars the H-contribution is non-negligible in its effect on $\mu$, which can 


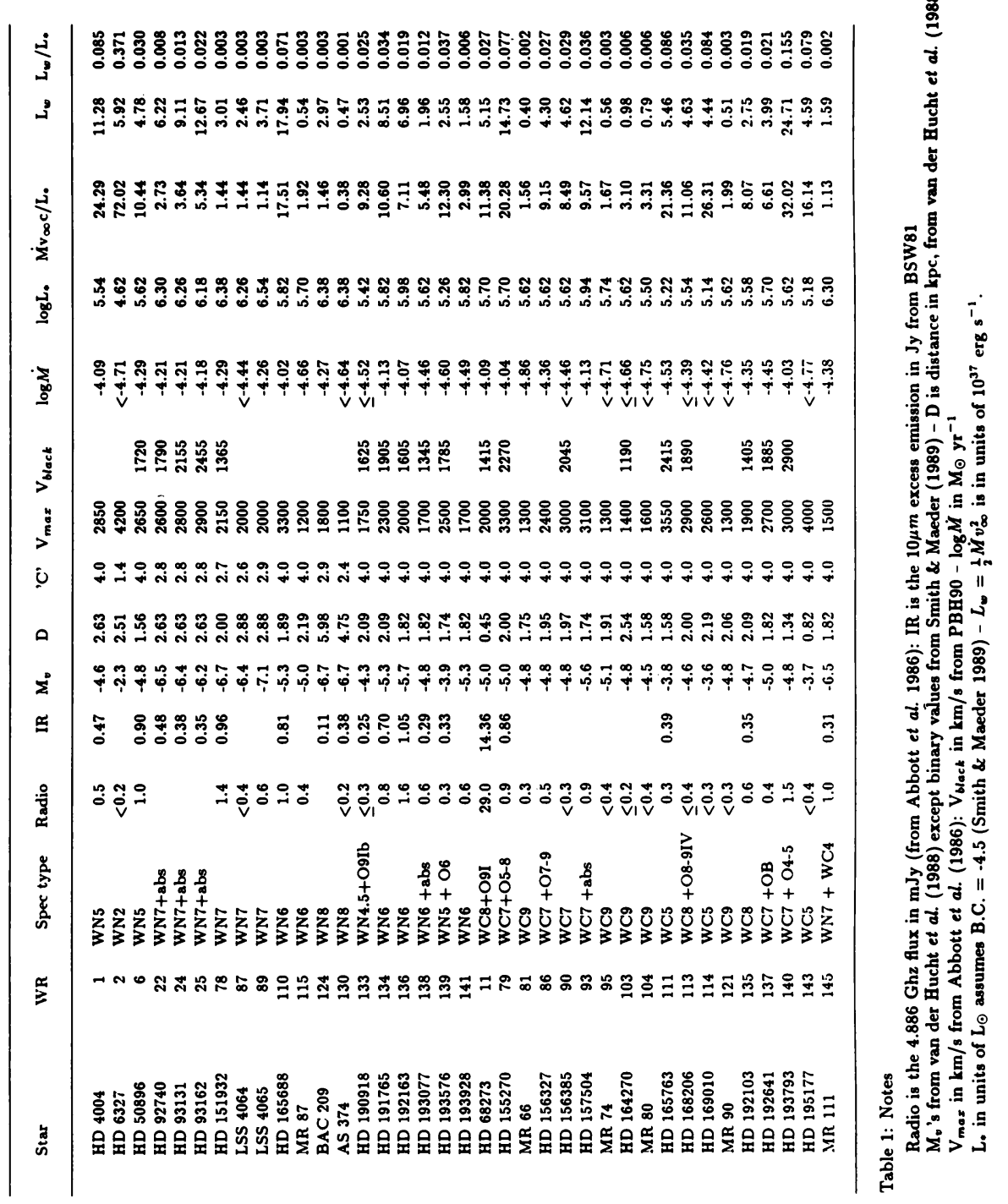


Fig 5: revised $\dot{M}$ vs. subtype - filled and open symbols are single and binary stars respectively

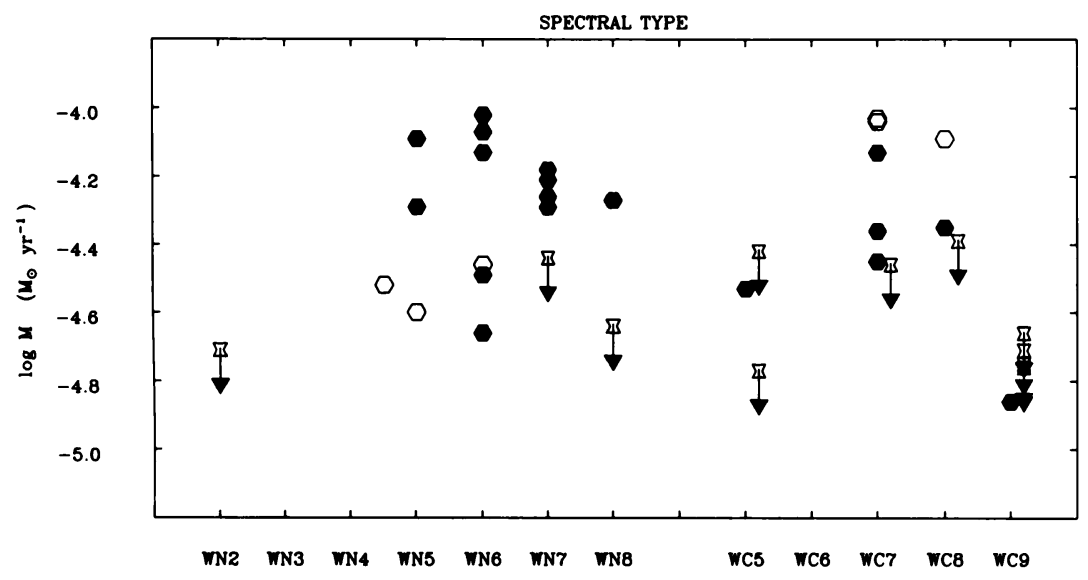

vary from $2.44-2.92, \mathrm{Z}=\gamma=1.0$, and thus $C$ lies in the range $2.44-2.92$, depending on the H-content given by Conti, Leep \& Perry (1983). (N.B. if $\mathrm{He}^{++}$were the dominant He ion in the radio region, the corresponding $C$-values would be: 1.41 (WNE) and 1.29 1.39 (WNL)). For WC stars, the most recent estimates of the $\mathrm{C} / \mathrm{He}$ ratios have been given by Smith \& Hummer (1988) from analyses of near-infared recombination lines. They find $\mathrm{C} / \mathrm{He}=0.3$ for WC6-9 stars; $=0.5$ for WC5 stars and $=0.7$ for WC4 stars. With He ${ }^{+}$as the dominant He ion in the radio region, (Smith \& Hummer confirm this in general), then the $C$-value depends only on the presumed carbon ionisation balance. Adopting $\mathrm{C}^{2+}$ as dominant leads to $C$-values of $\sim 4$ for all WC subclasses (increases in $\mu$ from 5.85 (WC6-9) to 7.30 (WC4) are compensated by corresponding increases in $\mathrm{Z}$ and $\gamma$ ). (N.B. for $\mathrm{He}^{++}$ dominant, $C$-values would be $\sim 2$ ). The $C$-values adopted herein, based on the above discussion are also listed in Table 1.

\section{Radio mass loss rates}

Table 1 presents revised mass loss rates for galactic WR stars, confirmed as thermal sources, taking into account revisions to the $C$-factors and $\mathrm{v}_{\infty}$ since the work of $\mathrm{ABCT}$, discussed above. Distances are taken from van der Hucht et al. (1988). Mass loss rates have been calculated using the radio $4.9 \mathrm{GHz}$ fluxes or upper limits, or (for stars with no radio observations) by predicting $4.9 \mathrm{GHz}$ fluxes by scaling the excess $10 \mu \mathrm{m}$ data using a spectral index of 0.75 . The terminal velocity is taken as $v_{b l a c k}$ from $P B H$ or as $0.74 \times v_{\max }$.

The WR mass loss rates lie in the range $10^{-5}-10^{-4} \mathrm{M}_{\odot} \mathrm{yr}^{-1}$, and are plotted as a function of WN and WC subtype in Fig 5. The overall mean value (excluding upper limits) is $5.3 \pm 2.3 \times 10^{-5} \mathrm{M}_{\odot} \mathrm{yr}^{-1}$, with no significance difference between this value and the means for (a) single or binary stars, (b) WN or WC stars. (c) WNE or WNL, (d) WCE or WCL stars. The apparently similarity in $\dot{M}$ between WN and WC stars, and between WCE and WCL stars is open to uncertainty, given the assumptions about the carbon ionisation balance in the radio-emitting regions. ABCT, BSW and van der Hucht et al. (1986) have argued that $\dot{M}$ for WC stars may be systematically larger (by $\sim \times 2)$ than for WN stars. 


\section{Mass loss rates - other methods}

Other independent methods of deriving mass loss rates for WR stars are generally based on studies of WR+O binary systems. For V444 Cyg, Khaliullin et al. (1984) have measured the period change of the system (using data over 1902-1983) to be $\dot{P}=0.22$ $\pm 0.04 \mathrm{sec}_{\mathrm{yr}^{-1}}$, which modelled in terms of a spherically symmetric outflow beyond the system leads to a mass loss rate determination of $\dot{M}=1.1 \times 10^{-5} \mathrm{M}_{\odot} \mathrm{y}^{-1}$.

St-Louis et al. (1988) have studied the phase-dependent variation in optical linear polarisation for $10 \mathrm{WR}+\mathrm{O}$ systems, which they interpret in terms of scattering of the companion star light off free-electrons in the WR wind to derive a new, independent method of calculating WR mass loss rates in such systems. The polarisation data also yields values for the individual orbit inclinations and thus stellar masses. Their results, shown in Fig 6, give values of $\dot{M}$ in the range $10^{-4}-10^{-5} \mathrm{M}_{\odot} \mathrm{y}^{-1}$, comparable in scale to that derived from the radio-IR data.

In the case of both $\mathrm{WN}+\mathrm{O}$ and $\mathrm{WC}+\mathrm{O}$ binaries, strong phase-dependent variability is also a common property in the ultraviolet - even for systems which show no optical eclipses. Pronounced line profile changes in the UV resonance and low-excitation lines are seen interpreted in terms of selective line-eclipses of the O-star light as it shines through the WR wind (Willis \& Wilson 1976, Koenigsberger \& Auer 1985). Such eclipse effects in the CIII] $\lambda 1909$ line in $\gamma$ Velorum (Willis et al. 1979), and in CV Serpentis (Howarth et al. 1982) have been modelled to yield mass loss rates for the WC8 components in these two systems of $9 \times 10^{-5}$, and $7 \times 10^{-5} \mathrm{M}_{\odot} \mathrm{y}^{-1}$ respectively.

Additionally, the detailed model atmosphere analyses of the helium line spectra of $\mathbf{3 0}$ galactic WR stars by Schmutz et al. (1989) provide estimates of mass loss rates for individual stars, yielding values in the range $10^{-5.3}-10^{-3.9} \mathrm{M}_{\odot} \mathrm{yr}^{-1}$, in excellent agreement with results from the radio data.

\section{7. $\dot{M}:$ L relations \& the wind momentum problem}

It is now well established that for OB stars $\dot{M} \propto \mathrm{L}^{1.6}$ (ABCT, Howarth \& Prinja 1989 ), and that this observed relation agrees well with predictions of radiation pressure mass loss theory. Attempts to ascertain a corresponding scaling for WR stars are plagued by uncertainties in the luminosities to be applied, given that the values for $\mathrm{T}_{\text {eff }}$ and bolometric corrections are very uncertain for this stellar class.

$A B C T$ found for five WR+O double-lined systems a tentative relation between WR mass loss rate and mass of the form $\dot{M} \sim 7 \times 10^{-8}\left(\mathrm{M} / \mathrm{M}_{\odot}\right)^{2.3} \mathrm{M}_{\odot} \mathrm{yr}^{-1}$, which, together with the Maeder $(1983)$ WR mass-luminosity relation $\left(\log \left(\mathrm{L} / \mathrm{L}_{\odot}\right)=3.8+1.5 \log \left(\mathrm{M} / \mathrm{M}_{\odot}\right)\right)$ implies $\dot{M} \sim 7 \times 10^{-14}\left(\mathrm{~L} / \mathrm{L}_{\odot}\right)^{1.6}$ (similar in slope to that for OB stars). They further deduced a rough relation between $\dot{M}$ and the quantity $\mathrm{M}_{v}-\left\langle\mathrm{M}_{v}\right\rangle$ (the difference for an individual WR star between its measured $M_{v}$ determined from cluster membership and the average value for its spectral type), providing additional support to the assertion of a WR mass loss rate - luminosity relation.

Smith \& Maeder (1989) used new data on galactic WR stellar masses and values of $\mathrm{M}_{v}$, to show (Fig 7) that for both WN and WC stars in WR+O binary systems, a reasonably well-defined relation exists in $\mathrm{M}_{v}$ vs. $M_{W R}$, which has the same slope as that predicted theoretically for stellar evolution models for He-burning stars with initial masses between 120 and $40 \mathrm{M}_{\odot}$, from Maeder \& Meynet $(1987)\left(\log \left(\mathrm{L} / \mathrm{L}_{\odot}\right)=4.02+\right.$ $\left.1.34 \log \left(\mathrm{M} / \mathrm{M}_{\odot}\right)\right)$. This comparison yielded a B.C. of $-4.5 \mathrm{mag}$., which they suggest can be applied to all WR subclasses. Applying this B.C. for stars with known $\mathrm{M}_{v}$ and mass loss 
Fig 6: $\dot{M}$ vs. $M_{W R}$ from polarisation studies of $W R+O$ systems (from St-Louis et al. 1988)

Fig 7: $M_{v}$ vs. $M_{W R}$ for WR binaries from Smith $\&$ Maeder (1989) implying a B.C. $=-4.5$ mag when compared to the evolutionary models of Maeder $\&$ Meynet (1987).
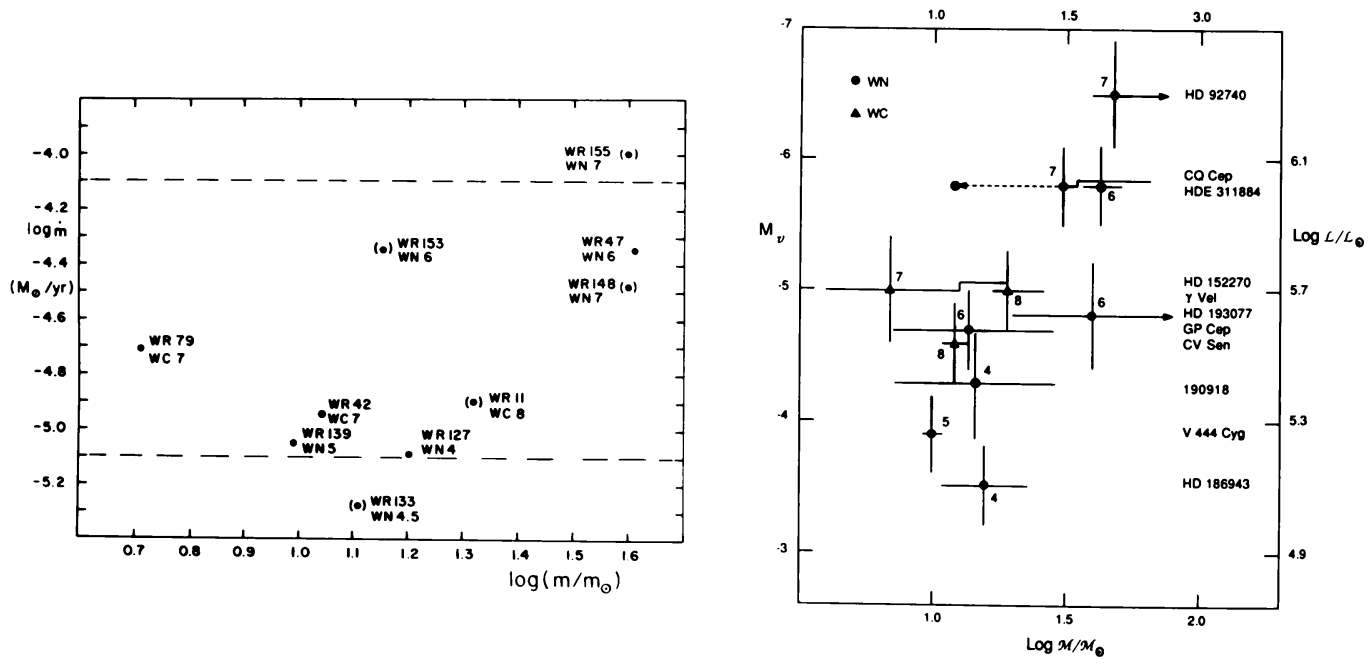

rates from radio and/or IR data from ABCT and BSW, they deduce a rough scaling of $\dot{M} \propto \mathrm{L}^{0.7}$, with a spread in $\dot{M}$ for any given $\mathrm{L}$ of an order of magnitude (see Fig 8 ). The slope is considerably lower than the value of 1.6 deduced by ABCT. The revised values of $\dot{M}$ given in Table 1 , plotted against $\mathrm{L}$, would not alter their conclusions. The results from St-Louis et al. (1988) (see Fig 6) indicate a rough scaling with $\dot{M} \propto \mathrm{M}_{W R}{ }^{0.8-1.3}$, comparable to the Smith \& Maeder (1989) conclusions. At present therefore, available data would suggest a rough scaling of WR mass loss with radiative luminosity: $\dot{M}(\mathrm{WR})$ $\propto \mathrm{L}^{\sim 1}$, shallower than for OB stars, and with a spread of up to an order of magnitude.

For WR stars characterising the ratio of the momentum in the wind to the single scattering radition pressure limit, viz. $\dot{M} v_{\infty} \mathrm{c} / \mathrm{L}_{*}$, again suffers from uncertainties in $\mathrm{L}_{*}$. Adopting a single B.C. $=-4.5$ mag for WR stars from Smith \& Maeder (1989) results in the values of $L_{*}$ given in Table 1 , which can probably be taken as upper limits to the radiative luminosities. The resulting momentum ratios, given in Table 1 , show values in the range $\sim 1-30$, with a trend for lower values for late-type WN and WC9 stars: mean values are: 2.2 (WN7,8), 10.3 (WNE), 13.5 (WC5-8) and 2.3 (WC9). These values are larger than appropriate for $\mathrm{OB}$ stars which typically have values $>1$. Adopting lower B.C's and L*'s proposed by BSW and Schmutz et al. (1989) will only exacerbate this problem, since then values of the momentum ratio would rise up to 50 or so. The results indicate a mechanism other than radiation pressure may be needed to initiate the WR mass loss, unless multiple scattering is extremely effective. Abbott (1982) concluded that chemical composition effects alone could not enhance the radiative driving for WN stars, and only by $\leq \times 3$ for WC stars, whilst attempts so far to include multiple scattering have led to gains of $\leq \times 5$ (e.g. Friend \& Castor 1983). Smith \& Maeder (1989) propose that (in addition to radiation pressure effects) an increase in mass loss rate might be promoted by mechanical instability due to the $\epsilon$-mechanism whose effect will be enhanced with changing mass and $\mu$ within the star. The expectation is thus that for WN7 and WN8 stars, which generally show appreciable atmospheric hydrogen, radiation pressure 
Fig 8: $\dot{M}$ vs. $L_{W R}$ from Smith \& Maeder (1989) assuming B.C. $=-4.5$ mag.

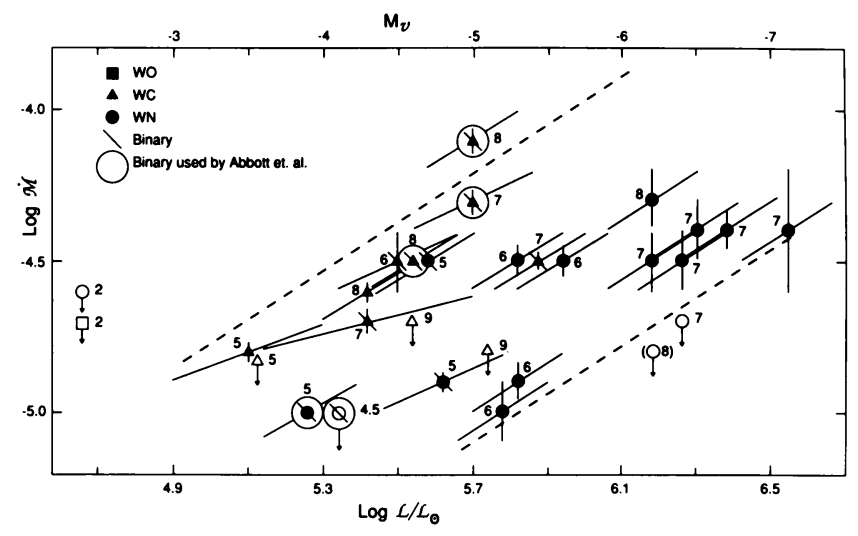

is probably the major driving mechanism. As the last of the hydrogen is removed, the instability mechanism is activated, and the mass loss rate enhanced. Alternatively, Poe, Friend \& Cassinelli (1989) have developed an axisymmetric model for WR winds involving a slow-dense equatorial flow and a fast, radiation-driven wind at higher latitudes, with high rotation ( $\sim 85$ percent maximum) and substantial, open magnetic fields $(\sim 1500 \mathrm{G})$ needed to accommodate the observed radio data and high wind speeds. The theory of WR winds and mass loss will be addressed further by Cassinelli (these proceedings).

Whatever mechanism(s) is driving the WR mass loss it is clear that these stars are a major source of mechanical energy input (and chemical enrichment) into the interstellar medium. Using the results for WR stars within $3 \mathrm{kpc}$ of the Sun, this topic has been discussed by ABCT and updated by van der Hucht et al. (1986) who give the following inputs to the ISM: wind mass flux $=1.6 \times 10^{-4} \dot{M} \mathrm{yr}^{-1} \mathrm{kpc}^{-2}$, wind momentum $\left(\dot{M} v_{\infty}\right)$ $=2.3 \times 10^{30} \mathrm{~g} \mathrm{~cm} \mathrm{~s}^{-2} \mathrm{kpc}^{-2}:$ and wind energy input $=3.2 \times 10^{38} \mathrm{erg} \mathrm{s}^{-1} \mathrm{kpc}^{-2}$, noting that whilst for WR+OBA stars, the former supply only $\sim 8 \%$ of the input radiative luminosity, they supply $\sim 70 \%$ of the mechanical mass, momentum and energy input. The effects of these inputs will be discussed elsewhere at this meeting.

\section{WR wind instability}

In addition to variability that can clearly be ascribed to phase-dependent effects in $\mathrm{WR}+\mathrm{O}$ binary systems, it is becoming increasingly clear that the stellar winds of WR stars are intrinsically variable. Single WR stars often show optical continuum light variability at the level of $\sigma_{\nu} \sim 0.003-0.03 \mathrm{mag}$. (Lamontagne \& Moffat 1987), as well as continuum polarisation varaibility at a level of $\sigma_{p} \sim 0.01-0.15 \%$ (Robert et al. 1988). High precision optical spectroscopy in the He II $\lambda 5411$ line of HD 191765 (WN6) presented by Moffat et al. (1988) reveal time-dependent structure (emission bumps) in the emission profile, which appear to accelerate outward along with the ambient wind on a time-scale of hours. Moffat et al. (1988) interpret these data in terms of rapid ejections of material condensations ('blobs').

In the ultraviolet, IUE spectroscopy has provided strong evidence for intrinsic WR line profile variability particularly evident in the $\mathrm{P}$ Cygni absorption components. Results for HD 50896 (WN5) indicate a variability timescale of $\sim 1$ day (Willis et al. 1989, St-Louis 
et al. 1990), with a similar timescale for HD 192163 (WN6) (St-Louis et al. 1989). For HD 96548 (WN8) a longer timescale of UV variability of $\sim$ several days seems present (Smith et al. 1986). Both the UV and optical data indicate that the amplitude of this shorttimescale variability can alter with epoch - for instance long term photometric monitoring of HD 50896 shows extended periods of relative 'inactivity', with abrupt changes to higher levels of variation (van der Hucht et al. 1990). Similarly, IUE monitoring has shown that HD 50896 can undergo periods of stability in its UV line profiles (Willis et al. 1989).

Whether or not radiation pressure is the mechanism initiating the higher levels of WR mass loss, it is likely that radiation pressure dominates the acceleration in their winds. It is now recognised that the radiation-driven winds of $O B$ stars are highly unstable and develop strong reverse shocks (Owocki, Castor \& Rybicki 1989) which may provide a natural explanation for their observed X-ray emission, extended blackness in the absorption components of UV resonance line $\mathrm{P}$ Cygni profiles, and the occurence and variability in discrete absorption components seen in these lines (Howarth \& Prinja 1989). The WR UV $P$ Cygni profiles also show extended regions of blackness; the X-ray emission from WR stars is (generally) comparable to that of OB stars (Pollock 1987), and therefore the WR winds may also be permeated by strong shocks, with the intrinsic WR wind variability caused by the same mechanism that generate the wind instabilities in the OB stars, with a greater amplitude reflecting enhanced WR wind densities.

\section{WR mass loss in other galaxies}

OB stars in the LMC and SMC show significantly lower stellar wind terminal velocities, and (possibly) lower mass loss rates from studies of their UV resonance line P Cygni profiles (e.g. Prinja 1987, Garmany \& Fitzpatrick 1988), which is broadly in line with expectations of radiation pressure-driven wind models for lower metallicity environments. Whilst no attempts have been made to actually determine mass loss rates for WR stars in the MCs, its appears from the overall nature of their optical and UV spectra that there may be no significant differences in $\dot{M}$ and $v_{\infty}$ with galactic counterparts. Estimates of $v_{\infty}$ for LMC stars, both from IUE P Cygni profile data and optical line widths, agree with galactic values of corresponding subtypes (Abbott \& Conti 1987). Smith et al. (1990) find a tight correlation between the ratio of the widths of $\lambda 5808 / \lambda 4650$ and the $\lambda 5808$ line width itself which is the same for both LMC and galactic WC stars.

The strength of the optical emission line spectrum may be taken as indicative of the mass loss rate ( $c f$ the optical emission measure vs. radio flux correlation from $A B C T$ ). Conti \& Massey (1989) find that the line strengths of leading optical WN and WC emission lines are very similar in the LMC and the Galaxy, whilst Conti, Massey \& Garmany (1990) suggest that the optical helium and nitrogen lines in the WNE stars in the SMC may be systematically weaker in strength (but not in velocity width) than galactic counterparts, although here the very small number of stars and binary companion effects may be playing a role. Further afield, Massey et al. (1987) find that WN and WC stars in M33 show similar optical line strength vs. line width correlations as found for galactic counterparts, whilst the few WR stars in M31 studied show poorer agrement. On balance, available UV and optical data do not point to gross mass loss or wind velocity differences between extragalactic and galactic WR stars of the same subtypes. What is striking, of course, is the substantial differences between the total WR populations and subtype occurence in the different galactic environments. If differing global galaxy metallicity is playing a role with regard to WR stars, its effect is possibly more pronounced in the WR precursor phase, than in significantly modifying the mass loss and winds of WR stars once they have formed. 


\section{References}

Abbott, D.C., 1982, Proc IAU Symp. No.99, (eds C. de Loore \& A.J. Willis), D. Reidel, p 185

Abbott, D.C., Bieging, J.H., Churchwell, E., \& Torres, A.V., 1986, Astrophys. J., 303, 239

Abbott, D.C., \& Conti,P.S., 1987, Ann. Rev. Ast. Astrophys., 25, 113

Barlow, M.J., 1982, Proc IAU Symp. No.99, (eds C de Loore \& A J Willis), D. Reidel, p 149

Barlow, M.J., Smith, L.J., \& Willis, A.J., 1981, Mon. Not. R. astr. Soc., 196, 101

Barlow, M.J., Roche, P.F., \& Aitken, D.K., 1988, Mon. Not. R. astr. Soc., 232, 821

Becker, R.H., \& White, R.L., 1985, Astrophys. J., 297, 649

Cherepaschuk, A.M., Eaton, J.A., \& Khaliullin, Kh.F., 1984, Astrophys. J., 281, 774

Conti, P.S., Leep, E.M., \& Perry, D., 1983, Astrophys. J., 268, 228

Conti, P.S., \& Massey, P., 1989, Astrophys. J., 337, 251

Conti, P.S., Massey, P, \& Garmany, C.D., 1990, Astrophys. J., 341, 113

Dickel, H.R., Habing, H.J., Isaacman,R., 1980, Astrophys. J. Lett., 238, L39

Florkowski, D.R., 1982, IAU Symposium No. 99, (eds C de Loore \& A J Willis), D. Reidel, p 63

Friend, D.B., \& Castor, J.I., 1983, Astrophys. J., 272, 259

Friend, D.B., \& Abbott, D.C., 1986, Astrophys. J., 311, 701

Garmany, C.D., \& Fitzpatrick, E.L., 1988, Astrophys. J., 332, 711

Hamann, W-R., 1981, Astr. Astrophys., 93, 353

Hamann, W-R., Schmutz, W., \& Wessolowski, U., 1988, Astr. Astrophys., 194, 190

Hillier, D.J., 1987, Astrophys. J. Suppl., 63, 965

Hillier, D.J., 1989, Astrophys. J., 347, 392

Hogg, D.E., 1982, IAU Symp. No. 99, (eds C de Loore \& A J Willis), D. Reidel, p 221

Hogg, D.E., 1985, in Radio Stars, (eds R Hjellming \& O Gibson), D.Reidel, p 117

Howarth, I.D., Willis, A.J., \& Stickland, D.J., 1982, ESA SP-176, p 331

Howarth, I.D., \& Prinja, R.K., 1989, Astrophys. J. Suppl., 69, 527

Hummer, D.G., Barlow, M.J., \& Storey, P.J., 1982, IAU Symp. No. 99, (eds C de Loore \& A J Willis), D.Reidel, p 79

Jones, P.A., 1985, Mon. Not. R. astr. Soc., 216, 613

Khaliullin, Kh.F., Khaliullina, A.I., \& Cherepaschuk, A.M., 1984, Pis'ma Astron. Zh., 10, 600 (= Sov. Astron. Letter., 10, 250)

Koenigsberger, G., \& Auer, L.H., 1985, Astrophys. J., 297, 255

Kuhi, L.V., 1973, Proc IAU Symp. No. 49, (eds M Bappu \& J Sahade), D.Reidel, p 205

Lamontagne, R.L., \& Moffat, A.F.J., 1987, Astr. J., 94, 1008

Lucy, L.B., 1982, Astrophys. J., 255, 278

Lucy, L.B., 1983, Astrophys. J., 274, 372

Maeder, A., 1983, Astr. Astrophys., 120, 113

Maeder, A., \& Meynet, G., 1987, Astr. Astrophys., 182, 243

Massey, P., Conti, P.S., \& Armandroff, T.E., 1987, Astr. J., 94, 1538

Moffat, A.F.J., Drissen, L., Lamontagne, R.L., \& Robert, C., 1988, Astrophys. J., 334, 1038

Owocki, S., Castor, J.I., \& Rybicki, G.B., 1988, Astrophys. J., 335, 914

Pauldrach, A., Puls, J., \& Kudritzki, R.P., 1986, Astr. Astrophys., 164, 86

Poe, C.H., Friend, D.B., \& Cassinelli, J.P., 1989, Astrophys. J., 337, 888

Pollock, A.M.T., 1987, Astrophys. J., 320, 283

Prinja, R.K., 1987, Mon. Not. R. astr. Soc., 228, 173

Prinja, R.K., Barlow, M.J., \& Howarth, I.D., 1990, Astrophys. J., 361, in press 
Purton, C.R., Fieldman, P.A., Marsh, K.A., Allen, D.A., \& Wright, A.E., 1982, Mon. Not. R. astr. Soc., 198, 321

Robert, C., Moffat, A.F.J., Basten, P., Drissen, L., \& St-Louis, N., 1989, Astrophys. J., 347, 1034

Schmutz, W., Hamann, W-R., \& Wessolowski, U., 1989, Astr. Astrophys., 210, 236

Smith, L.F., \& Hummer, D.G., 1988, Mon. Not. R. astr. Soc., 230, 511

Smith, L.F., \& Maeder, A., 1989, Astr. Astrophys., 211, 71

Smith, L.F., Shara, M.M., \& Moffat, A.F.J., 1990, Astrophys. J., 348, 471

Smith, L.J., Willis, A.J., Garmany, C.D., \& Conti, P.S., 1986, ESA SP-263, p 389

St-Louis, N., Moffat, A.F.J., Drissen, L., Bastien, P., \& Robert, C., 1988, Astrophys. J., 330, 286

St-Louis, N., Smith, L.J., Stevens, I.R., Willis, A.J., Garmany, C.D., \& Conti, P.S., 1989, Astr. Astrophys., 226, 249

St-Louis, N., Smith, L.J., Willis, A.J., Garmany, C.D., \& Conti, P.S., 1990, ESA SP-310, in press

Torres, A.V., Conti, P.S., \& Massey, P., 1986, Astrophys. J., 300, 379

van der Hucht, K.A., Cassinelli, J.P., \& Williams, P.M., 1986, Astr. Astrophys., 168, 111

van der Hucht, K.A., Hidayat,B., Admironto, A.G., Supelli, K.R., \& Doom, C., 1988, Astr. Astrophys., 199, 217

van der Hucht, K.A., van Genderen, A.M., \& Bakker, P.R., 1990, Astr. Astrophys., 228, 108

Williams, P.M., Eenens, P.R.J., 1989, Mon. Not. R. astr. Soc., 240, 445

Williams, P.M., van der Hucht, K.A., Sondell, G., \& The, P.S., 1990, Mon. Not. R. astr. Soc., 244, 101

Willis, A.J., 1982, Mon. Not. R. astr. Soc., 198, 897

Willis, A.J., \& Wilson, R., 1976, Astr. Astrophys., 47, 429

Willis, A.J., et al., 1979, The 1st Year of IUE (UCL), p 394

Willis, A.J., Howarth, I.D., Smith, L.J., Garmany, C.D., \& Conti, P.S., 1989, Astr. Astrophys. Suppl., 77, 269

Wright, A.E., \& Barlow, M.J., 1975, Mon. Not. R. astr. Soc., 170, 41 


\section{DISCUSSION}

Smith, Lindsey: As co-author of the quoted low power of $\dot{M}$ dependence on $M($ or $L)$, I should point out that the other author, Maeder, is now using $\dot{M} \propto M^{2.5}$ (suggested by Langer) with spectacular success. Also remembering that Langer suggests a higher rate for WC than WN, it looks like the stars, as they evolve zig-zag across the observed band in the $\dot{M}-L$ diagram.

Willis: I am aware that the stellar evolutionists are employing a steeper $\dot{M}$ vs. $M$ relation than implied by your work and that from the polarization results. My remit was to concentrate on observational results, which at the present do not necessarily support the theoretical viewpoint directly. I cannot help that, but there may still be considerable uncertainties in both $M_{W R}$ and $L$ rather than in mass loss rate, which is masking the true observational picture.

Conti: Why do we not find a single $\dot{M}-L$ relation for WR stars? After all, there is one for $\mathrm{O}$ stars. The stellar structure people predict a $\dot{M}$-mass relation. We believe $\mathrm{W}-\mathrm{R}$ winds are radiatively driven. Could one instead assume there is a unique (displaced from the $\mathrm{O}$ star one due to chemistry) $\dot{M}-L$ relation for WR stars and use this to infer the WR stars $L$ (and B.C.) since the $\dot{M}$ seems well known?

Willis: Again, the recent results in the literature based on observations of mass loss rate, $L$ and $\dot{M}_{W R}$, do not point to a well-defined $\dot{M}-L$ relation for WR stars, nor one with the same slope as for OB stars. Whilst radiation pressure is undoubtedly going to be important in WR winds, it may not be the only mechanism driving their mass loss. In that event a simple relation with $L$ may not necessarily be expected. On the other hand, I agree it would be interesting to see the implications of assuming a relation that you propose on the inferred $L$ and $\dot{M}_{W R}$.

Sreenivasan: Questioning observations is supposed to be in bad taste. You say that mass loss rates are uncorrelated with spectral type but Langer says he finds good agreement with observations if he uses a mass loss rate $\propto M^{2.3-2.5}$. This is confusing. Is it still in bad taste to question the observations and/or the interpretations? (From a theoretical point of view, radiatively driven winds give mass loss rates proportional to a power of luminosity and there definitely are other sources/mechanisms of mass loss in WR stars.).

Willis: I think the observations (radio, IR, polarization and UV data) are in pretty good shape, but the interpretation is still subject to some uncertainty. For instance modelling the radio emission does require a knowledge of the ionization balance (and chemistry) in the radio region, which at least for WC stars is not too well known. Factors of two or so uncertainty cannot be ruled out, say between WCE and WCL stars. As for other mechanisms driving WR winds, I can fortunately defer that aspect to Joe Cassinelli in the next review talk!

Pakull: You mentioned bolometric corrections of about -4 to -4.5 .

Willis: -4.5 .

Pakull: I will tell you tomorrow that there are some WR stars, namely WN, very early ones, which have bolometric corrections of about -7 . These are very very hot stars which also ionize large $H e I I I$ regions around them and in these stars you also have of course in a radio-emitting region $\mathrm{He}$ completely ionized. And this may be a reason why you find for the WN2 stars very low $\dot{M}$ whereas this is only because the $\mathrm{He}$ is completely ionized also in the radio-emitting region.

Willis: As far as the bolometric correction is concerned, I was attempting to illustrate the mass loss rate momentum problem by picking a luminosity which I felt was probably an 
upper limit. I took the Smith-Maeder results, partly because I knew Lindsey Smith was chairing this session. For the bulk of the stars, the Schmutz values alone already raise the momentum question. So, it was an attempt to summarize the lower level of the problem, if a problem it is, and of course we have the bolometric correction problem... If you have -7 , which I have not come across before I might add, that is going to ease it considerably. But, -7 will not apply to the bulk of the WR subclasses quite demonstratively, it is not going to affect the momentum ratio problem in general.

Schmutz: First thing we did after analyzing 30 stars, was looking at the mass loss rate and the luminosity and at the moment we consider our models to be good within 1 magnitude for the bolometric corrections, as absolute. But by doing so, comparing stars which have been analyzed by the same methods, you actually have problably the same systematic errors for all. So, your trend would show up much easier, and there simply is no trend of mass loss rate with luminosity. I do not believe there is any.

Moffat: A more recent compilation of polarization data of $\mathrm{WR}+\mathrm{O}$ binaries in the Galaxy and the Magellanic Clouds ( $c f$. poster at this meeting) show that $\dot{M}_{W R}$ and $M_{W R}$ correlate rather well with spectral subclass for WN and WC sequences, with $\dot{M}_{W R}$ and $M_{W R}$. Possibly the radio $\dot{M}$ 's are inflicted with large scatter due to uncertainties in the distances which are not needed for the polarization $\dot{M}$ 's.

Willis: This is an interesting and important result that we need to consider. I am impressed with the utilization of polarization variability in constraining mass loss rates and relations with $M_{W R}$ and subtype.

Smith, Lindsey: A thing that should be pointed out is that the success of the models using Langer's mass loss rate $\propto M^{2.5}$ is pretty impressive, as I am sure we will hear more about in the future.

Underhill: Just to remind you again about V444 Cyg. Fourty years of photo-electric observations saw a very distinct rate of period change. Rate of period change is supposed to be connected with a rather simple formula to rate of mass loss from the system, from one star as I should say, actually both stars got winds. The momentum rate of mass loss you can get from your observed rate of period change is $6 \times 10^{-6} M_{\odot} y r^{-1}$. I think that it is a more fundamental way of estimating rate of mass loss than using the radio flux and saying that it is free-free in a spherical distribution. The fact that you got an index of 0.6 to 0.7 only means that, if it is free-free, you are observing a body of gas with a density gradient in it. It does not tell you that it is a sphere going out. That radio flux could have three sources.

Willis: I might add actually, that the $10 \mu-6 \mathrm{~cm}$ spectral index for V444 Cyg is 0.52 , which is almost exactly what you would expect $(0.53)$ for a constant velocity isothermal flow between the IR and radio region. V444 Cyg is the only star in our (BSW, 1981) 10 $\mu$ sample that does this.

There seems to be some discrepancy between the actual period change, between Cherepashchuk and yourself, that does not worry me too much, actually. Essentially, the radio data agree with the polarization measurements in sofar as both interpretations give a mass loss rate of about $10^{-5}$. That would agree with the Cherepashchuk period change. It is a factor or two larger than your measurement of period change of $0.08 s \cdot y r^{-1}$.

Underhill: (???)

Willis: What I am trying to say is that the mass loss rate inferred from these other analyses is at the lower bound of the range that one gets from the WR stars as a bunch and frankly I never get worked up about factors of two; factors of ten yes, but not factors of two.

Vanbeveren (to Underhill): You contineously repeat your $\dot{P} / \mathrm{P}$ argument. However, according to me, you are not consistent with your own WR model. One can derive $\dot{M}$ from 
$\dot{P} / \mathrm{P}$ if the $\dot{M}$ is spherically symmetric by using an easy formula. However, when you have a large accretion disc around the WR star (as you propose) then this easy relation between $\dot{P} / \mathrm{P}$ and $\dot{M}$ disappears.

Willis: I should point again out that Underhill's measurement of $\dot{P} / \mathrm{P}$ for V444 Cyg is a factor or two lower than the measurement of Khaliullin et al. (1984). The latter would give very good agreement with the radio mass loss rate. The question of who is right about the period change is something for these authors to sort out, but I am not particularly concerned about a factor of two in this game. Moreover, as we know, the mass loss rate for V444 Cyg is amongst the lowest for the WR stars, and the momentum problem is still there for most objects in this class.

Schmutz: I would like to point out that V444 Cyg is not a typical WN5 WR star. "Normal" strong lined WN5 stars have different line profiles. The most important difference is that weak high ionization lines of V444 Cyg are very narrow, e.g. $N V \lambda 4940$, in contrast to the broad profiles of strong-lined single WN5 stars. This difference is not due to different $v_{\infty}$ since the $v_{\infty}$ 's are similar. The narrow lines of V444 Cyg indicate therefore that we see down to low wind velocities whereas we do not see slow moving material in "normal" WN5 stars. Therefore V444 Cyg has not a typical velocity law which is in line with its mass loss rate that is at the lower limit of the observed rates.

Willis: I agree with you that the mass loss rate of the WN5 component in V444 Cyg (as deduced from the period change and the radio data) lies at the lower bound for WR stars, $\sim 1 \times 10^{-5} M_{\odot} y r^{-1}$, and as such we can probably "see" further into its wind down to low velocity parts. The reason for quoting the Cherepashchuk et al. (1984) velocity law for V444 Cyg was that, as far as I know, it is the only one which has been observed somewhat directly. On another topic, I concentrated on using the B.C. $=-4.5 \mathrm{mag}$ from Smith \& Maeder (1989), rather than your rather lower values (generally around B.C. $=-3$ mag), partly because I wanted to emphasise effectively the lower limits to the momentum problem, and partly because I knew Lyndsey Smith would be chairing this session, and it seemed a wise and prudent thing to do!

Cherepashchuk: Perhaps one could try to take into account the cloud ragged structure of the WR wind. If the WR wind in the infrared and the radio region is cloudy and ragged we can obtain the same radio and IR flux for a lesser value of $\dot{M}$. It may help us to eliminate some discrepancies between $\dot{M}$ values obtained from IR and radio data and those obtained from period change in binary systems.

Schulte-Ladbeck: I just want to remind ourselves that we have heard yesterday and today that there are large-scale inhomogeneities in WR star winds: blobs, shocks, cloudlets, disk, and different effects seem to prevail in different stars. While we all agree that radiation pressure is the first order effect, would you not expect to find a large scatter in the $\dot{M}-L$ relation for this very reason?

Willis: I have emphasised the new observations of intrinsic variability in WR stellar winds, both from optical and UV data. It may be that much of this can potentially be related to radiatively-induced instabilities in the winds of the kind that Owocki has talked about. On the other hand, other effects may well be operating and contributing to the $\dot{M}-L$ scatter. 